\title{
Creencias irracionales en estudiantes de derecho de una universidad particular de Chiclayo, Perú
}

Blanca Mariana Rojas-Jiménez ${ }^{1,2, a}$, Julio Cesar Suarez-Luna ${ }^{1, a}$, Juan de Dios Alvarez-Julca 1,a

\section{RESUMEN}

Objetivos. Describir las creencias irracionales en estudiantes de derecho de una universidad particular de Chiclayo, Perú, año 2016. Material y métodos. Investigación de tipo descriptiva. El muestreo fue probabilístico estratificado y con estudiantes de ambos sexos, entre las edades de 16 y 40 años. Para la recolección de los datos se utilizó el Inventario de Conducta Racional de Shorkey y Whiteman válido y confiable en nuestra realidad psicosocial peruana. Resultados. En general, el $44 \%$ y $43 \%$ de estudiantes mostró creencias irracionales de sentimientos de culpa y grado de independencia, respectivamente. Las estudiantes presentaron $47 \%$ y $42 \%$ de sentimiento de culpa y grado de independencia, respectivamente. Asimismo, el sentimiento de culpa en estudiantes con y sin trabajo fue $54 \%$ y $40 \%$, respectivamente. Conclusiones. Estudiantes de derecho de una universidad privada de Chiclayo evidencian creencias irracionales; con predominancia de sentimientos de culpa (mujeres) y grado de independencia, y en varones culpabilidad (varones). Es necesario establecer estrategias de intervención para asistir a la población estudiantil en riesgo de creencias irracionales.

Palabras clave: Conducta, Culpa, Estudiantes (Fuente: DeCS-BIREME).

\section{Irrational beliefs in law students from a private university in Chiclayo, Peru}

\section{ABSTRACT}

Objectives. Describe the irrational beliefs in law students from a private university in Chiclayo, Peru, 2016. Material and methods. Descriptive research. The sampling was stratified probability and with students of both sexes, between the ages of 16 and 40 years. To collect the data, a valid and reliable Inventory of Rational Behavior of Shorkey and Whiteman was used in our Peruvian psychosocial reality. Results. Overall, $44 \%$ and $43 \%$ of students showed irrational beliefs of guilt feelings and degree of independence, respectively. The female students presented $47 \%$ and $42 \%$ of feeling of guilt and degree of independence, respectively. Likewise, the feeling of guilt in students with and without work was $54 \%$ and $40 \%$, respectively. Conclusions. Law students from a private university in Chiclayo demonstrate irrational beliefs; with predominance of feelings of guilt (women) and degree of independence, and in men guilt (men). It is necessary to establish intervention strategies to assist the student population at risk of irrational beliefs.

Keywords: Behavior, Guilt, Students (Source: MeSH-NLM)

\footnotetext{
${ }^{1}$ Universidad de San Martín de Porres-filial Norte. Chiclayo, Perú.

${ }^{2}$ Servicio de Psicología, Hospital Regional Lambayeque. Chiclayo, Perú

a Psicóloga (o).
} 


\section{INTRODUCCIÓN}

Los problemas psicológicos en estudiantes universitarios a nivel mundial han sido objeto de estudio de numerosas investigaciones que reportan índices de prevalencias de diversos trastornos y problemas relacionados a la salud mental. Un aspecto fundamental para la vulnerabilidad de las alteraciones psicológicas en estudiantes universitarios y otras personas estaría dado, según Riso (1), en las vulnerabilidades biológicas y cognitivas, contenidos esquemáticos prepotentes que incluyen creencias, actitudes y asunciones acerca de sí mismo y los otros, que predisponen a contraer distintos tipos de alteraciones.

Por otro lado, Clark et al. (2), afirman que en realidad lo que dispara la diátesis o vulnerabilidad individual, no es la mera congruencia informacional, si no la percepción que la persona tenga sobre el evento y el impacto de éste sobre el autoconcepto. La diátesis, por sí sola, no es definitiva, y sola la presencia del evento estresante tampoco, es la conjunción de ambas variables la que hace que el trastorno aparezca. Por lo tanto, existen factores estresantes y un perfil cognitivo predispuesto, muchas veces con creencias irracionales a una disposición de diversos tipos de trastornos psicológicos en los estudiantes y las personas. Estos aportes teóricos de los autores también se pueden evidenciar específicamente en los estudiantes universitarios, ya que a menudo estos están expuestos a un intenso estrés en su ambiente, ya sea por la tensión en el cumplimiento de tareas, exámenes, competencias estudiantiles, retos personales, etc.; que pueden predisponer a la aparición de la diátesis o los diversos problemas y trastornos de índole psicológico según su perfil cognitivo.

Con lo expuesto, también podemos evidenciar diversos estudios que relacionan a los problemas de salud psicológica en personas y estudiantes universitarios con la vulnerabilidad cognitiva y más específicamente con sus creencias irracionales subyacentes, debido a que el comportamiento irracional es el núcleo en la diátesis-estrés para las posteriores posibles perturbaciones en las personas. Según Ellis (3), el comportamiento irracional normalmente tiene varios aspectos: la persona cree que la irracionalidad se ajusta a los principios de la realidad; las personas que se aferran a la irracionalidad se denigran o no quieren aceptarse a sí mismos, bloquean seriamente el conseguir la clase de relaciones interpersonales que les gustaría tener; impide su trabajo lucrativo y alegre en algún quehacer productivo.

Un estudio realizado por Manjarres (4) en Ecuador pudo describir la relación entre las ideas irracionales y las conductas agresivas en los estudiantes de los primeros cursos de bachillerato de los colegios fiscales urbanos del Cantón Pelileo; donde observaron que el $100 \%$ de los estudiantes presentaron ideas irracionales, destacándose la tendencia a pensar que "Se debe sentir miedo o ansiedad ante cualquier cosa desconocida, incierta o potencialmente peligrosa" reflejada en un $24 \%$, y la creencia de que "Se necesita contar con algo más grande y más fuerte que uno mismo" en el $22 \%$.

Esta investigación es de vital importancia para conocer la salud psicológica de los estudiantes que tendrán a cargo la implementación de la justicia en los diferentes ámbitos de la sociedad (estudiantes de derecho). Los resultados de este estudio también beneficiaron a los directivos de la Escuela de Derecho en poseer un diagnóstico de las creencias irracionales en sus estudiantes y fomentar medidas de tutoría para lograr mayor creencias racionales en su autovalía y autoconcepto en los estudiantes, por lo tanto contribuir a su desarrollo integral.

Por tanto, la presente investigación buscó determinar las creencias irracionales en estudiantes de derecho, de una universidad particular de Chiclayo, durante el año 2016.

\section{MATERIAL Y MÉTODOS}

\section{Tipo y diseño de investigación}

Estudio transversal descriptivo y prospectivo.

\section{Población y muestra}

La población estuvo constituida por 317 alumnos de la Escuela de Derecho de la Universidad de San Martín de Porres, Chiclayo, Perú; en el año 2016. El tipo de muestreo fue probabilístico estratificado y contó con 174 alumnos, entre las edades de 17 a 35 años, distribuidos en 102 mujeres y 72 varones.

\section{Técnicas e instrumentos de recolección de datos}

El instrumento utilizado fue el Inventario de Conducta Racional de Shorkey y Whiteman (5) (1977), para evaluar las creencias irracionales. Así mismo Aguinaga (2012) (6) refiere que la validez y confiabilidad de la versión adaptada fue realizada en Perú por León y Lihana y consta de 37 ítems, divididos en 11 factores: Frustración, Exceso de Culpa, Perfeccionismo, Necesidad de Aprobación, Necesidad de Ayuda y Cuidados, Sentimiento de Culpa, Inercia y Evasión, Grado de Independencia, Aprobación, Ideas de Infortunio, Confianza en el control de las Emociones.

La aplicación del inventario se llevó a cabo en un ambiente tranquilo y los evaluados respondieron por sí mismos a los ítems de la prueba. Para evitar sesgos y engaños referentes al instrumento se enfatizó que no había respuestas correctas ni incorrectas.

\section{Análisis de datos}

Para el análisis de los datos se hizo uso de la estadística descriptiva, que consistió en recopilar, presentar y categorizar la información con el fin de poder realizar la cen- 
tralización y dispersión. Así mismo; los datos y gráficos se procesaron y se construyeron con la ayuda de los programas SPSS 19 y Excel 2010.

\section{Análisis éticos}

Para la ejecución del presente trabajo, fue necesaria la aprobación de la Unidad de Investigación del Programa de Psicología de la Universidad San Martín Filial Norte. Los resultados se mantuvieron en total confidencialidad y solo fueron ser utilizados para el propósito de estudio. La participación fue voluntaria a través de la firma de un consentimiento informado.

\section{RESULTADOS}

Se evidencia que las creencias irracionales con niveles predominantemente altos son el factor 6: sentimientos de culpa con $44 \%$ y factor 8: grado de independencia con $43 \%$ (Figura 1). En la figura 2 se observa que las creencias con nivel predominantemente alto en el sexo femenino son el factor 6: sentimientos de culpa con $47 \%$ y el factor 8: grado de indepedencia con $42 \%$. Asimismo, en el figura 3 se muestra que la creencia irracional con nivel predominantemente alto en el sexo masculino es el factor 6: sentimientos de culpa con $40 \%$.

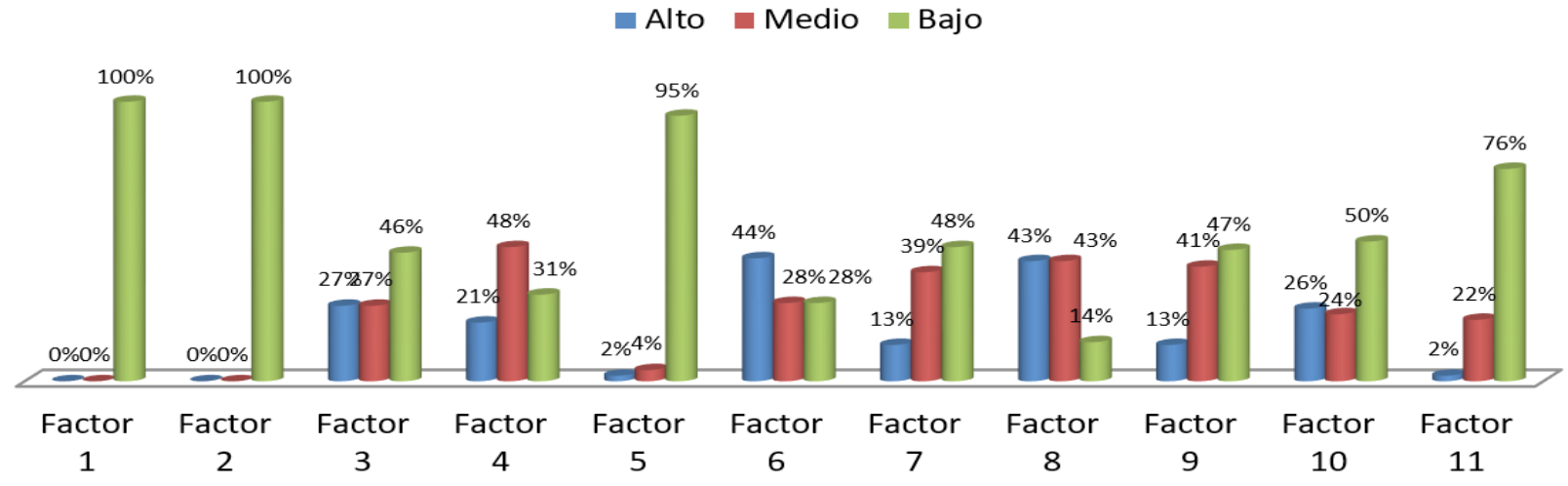

Figura 1. Creencias Irracionales en estudiantes de derecho de una universidad particular de Chiclayo, Perú, $2016 .$.

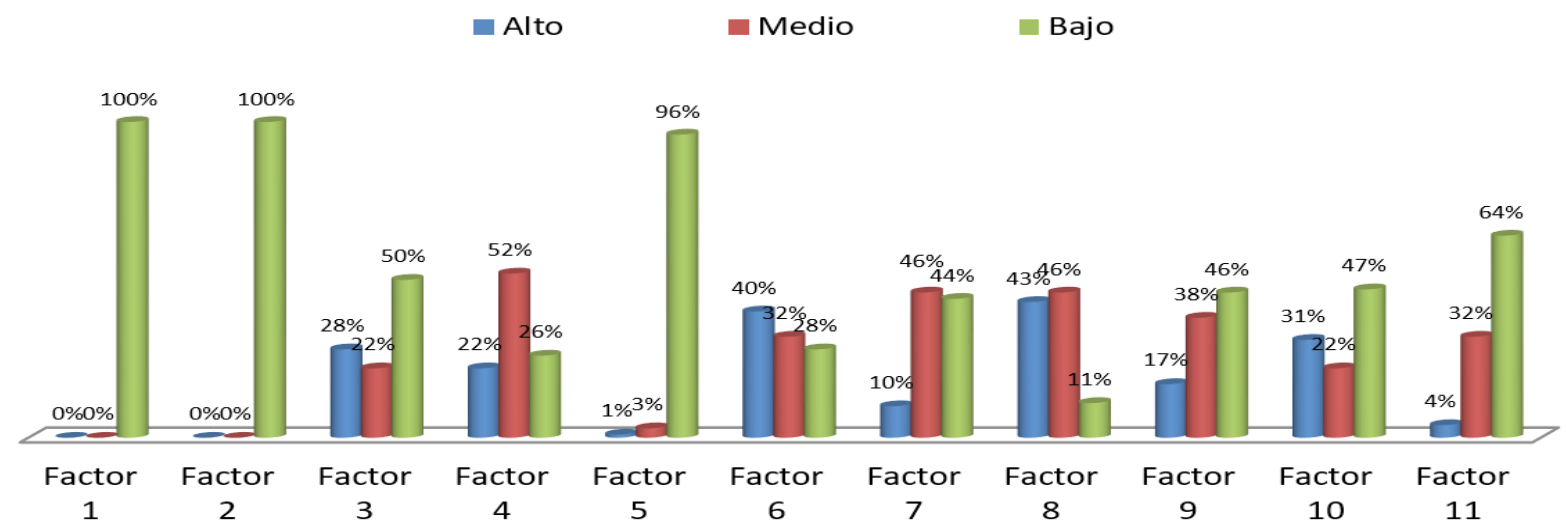

Figura 2. Creencias Irracionales según sexo femenino en estudiantes de derecho de una universidad particular de Chiclayo, Perú, 2016.

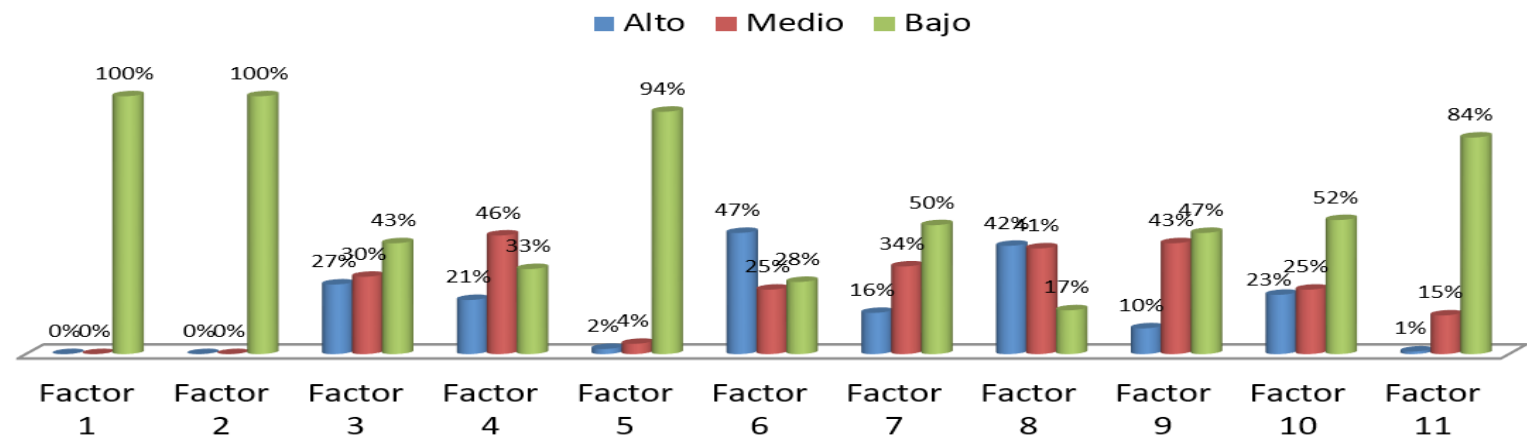

Figura 3. Creencias irracionales según sexo masculino en estudiantes de derecho de una universidad particular de Chiclayo, Perú, 2016. 
En la tabla 1, se evidencia que el nivel alto de creencias irracionales de los alumnos según la etapa de adolescencia se encuentra en el factor 6: sentimientos de culpa con $28 \%$. Por otro lado, los alumnos en la etapa de adultez temprana, obtuvieron un nivel alto de creencias irracionales en el factor 6: sentimientos de culpa y factor 8: grado de independencia con $16 \%$ cada uno.

Asimismo, en la tabla 2, se evidencia que el nivel alto de creencias irracionales en los alumnos que trabajan, se presentan en el factor 6: sentimientos de culpa con $54 \%$ y en el factor 8: grado de independencia con $46 \%$. Por otro lado, el nivel alto de creencias irracionales en los alumnos que no trabajan, se observa en el factor 6: sentimientos de culpa con $40 \%$.

Tabla 1. Creencias irracionales con nivel alto en estudiantes de derecho de una universidad particular de Chiclayo, 2016.

\begin{tabular}{|c|c|c|c|}
\hline Factores & Nivel & $\begin{array}{c}\text { Adolescencia } \\
(\%)\end{array}$ & $\begin{array}{c}\text { Adultez Tem } \\
\text { prana }(\%)\end{array}$ \\
\hline \multirow{3}{*}{ Factor 1} & Bajo & 65 & 35 \\
\hline & Medio & 0 & 0 \\
\hline & Alto & 0 & 0 \\
\hline \multirow{3}{*}{ Factor 2} & Bajo & 65 & 35 \\
\hline & Medio & 0 & 0 \\
\hline & Alto & 0 & 0 \\
\hline \multirow{3}{*}{ Factor 3} & Bajo & 32 & 14 \\
\hline & Medio & 16 & 12 \\
\hline & Alto & 18 & 9 \\
\hline \multirow{3}{*}{ Factor 4} & Bajo & 16 & 15 \\
\hline & Medio & 35 & 13 \\
\hline & Alto & 14 & 17 \\
\hline \multirow{3}{*}{ Factor 5} & Bajo & 60 & 35 \\
\hline & Medio & 3 & 1 \\
\hline & Alto & 2 & 0 \\
\hline \multirow{3}{*}{ Factor 6} & Bajo & 20 & 9 \\
\hline & Medio & 17 & 10 \\
\hline & Alto & 28 & 16 \\
\hline \multirow{3}{*}{ Factor 7} & Bajo & 30 & 18 \\
\hline & Medio & 26 & 13 \\
\hline & Alto & 9 & 5 \\
\hline \multirow{3}{*}{ Factor 8} & Bajo & 9 & 5 \\
\hline & Medio & 29 & 14 \\
\hline & Alto & 27 & 16 \\
\hline \multirow{3}{*}{ Factor 9} & Bajo & 33 & 14 \\
\hline & Medio & 26 & 14 \\
\hline & Alto & 6 & 7 \\
\hline \multirow{3}{*}{ Factor 10} & Bajo & 30 & 20 \\
\hline & Medio & 18 & 6 \\
\hline & Alto & 17 & 9 \\
\hline \multirow{3}{*}{ Factor 11} & Bajo & 49 & 26 \\
\hline & Medio & 14 & 8 \\
\hline & Alto & 2 & 1 \\
\hline
\end{tabular}

Tabla 2. Creencias irracionales en estudiantes de derecho de una universidad particular de Chiclayo, Perú, 2016; según condición de trabajo.

\begin{tabular}{|c|c|c|c|}
\hline Factores & Nivel & $\begin{array}{l}\text { Con Trabajo } \\
(\%)\end{array}$ & $\begin{array}{l}\text { Sin Trabajo } \\
(\%)\end{array}$ \\
\hline \multirow{3}{*}{ Factor 1} & Bajo & 100 & 100 \\
\hline & Medio & 0 & 0 \\
\hline & Alto & 0 & 0 \\
\hline \multirow{3}{*}{ Factor 2} & Bajo & 100 & 100 \\
\hline & Medio & 0 & 0 \\
\hline & Alto & 0 & 0 \\
\hline \multirow{3}{*}{ Factor 3} & Bajo & 46 & 46 \\
\hline & Medio & 30 & 26 \\
\hline & Alto & 24 & 28 \\
\hline \multirow{3}{*}{ Factor 4} & Bajo & 35 & 28 \\
\hline & Medio & 44 & 50 \\
\hline & Alto & 21 & 22 \\
\hline \multirow{3}{*}{ Factor 5} & Bajo & 96 & 94 \\
\hline & Medio & 4 & 3 \\
\hline & Alto & 0 & 3 \\
\hline \multirow{3}{*}{ Factor 6} & Bajo & 18 & 33 \\
\hline & Medio & 28 & 27 \\
\hline & Alto & 54 & 40 \\
\hline \multirow{3}{*}{ Factor 7} & Bajo & 42 & 50 \\
\hline & Medio & 39 & 39 \\
\hline & Alto & 19 & 11 \\
\hline \multirow{3}{*}{ Factor 8} & Bajo & 15 & 14 \\
\hline & Medio & 39 & 45 \\
\hline & Alto & 46 & 41 \\
\hline \multirow{3}{*}{ Factor 9} & Bajo & 42 & 49 \\
\hline & Medio & 40 & 41 \\
\hline & Alto & 18 & 10 \\
\hline \multirow{3}{*}{ Factor 10} & Bajo & 56 & 47 \\
\hline & Medio & 18 & 27 \\
\hline & Alto & 26 & 26 \\
\hline \multirow{3}{*}{ Factor 11} & Bajo & 67 & 80 \\
\hline & Medio & 30 & 18 \\
\hline & Alto & 3 & 2 \\
\hline
\end{tabular}

\section{DISCUSIÓN}

En los resultados se puede apreciar un nivel alto en las creencias irracionales de sentimiento de culpa y grado de independencia. Esto evidencia que el sentimiento de culpa predomina como creencia irracional, siendo una de las causantes de vulnerabilidad cognitiva para la baja autoestima y autoconcepto de un esquema propio disfuncional como lo refiere Riso (2009) (1). Esto puede repercutir en una pobre autoimagen y falta de aceptación personal en los estudiantes que merece ser tratado con programas de intervención psicológica.

Con respecto, a los factores de las creencias irracionales en un nivel alto, presentes en estudiantes de sexo feme- 
nino y masculino de la escuela de Derecho, se pudo evidenciar el de sentimiento de culpa. Díaz ${ }^{(8)}$, citado por Aguinaga (6) (2012) define al sentimiento de culpa como: pena del yo, culpa y por errores, pecados e injusticias. Dando a entender que los alumnos de ambos sexos poseen un grado alto de imperativos sobre una conducta moralmente aceptada en la sociedad, ceñida a reglas y normas que crean estándares o patrones de comportamiento, que ellos mismos observan como deteriorados o desvalorizados por no cumplir las expectativas referentes a su persona. Se debe señalar también, que estas creencias son irracionales y no se ajustan a la realidad. Es así, como un principio rector de las creencias irracionales en el sentimiento de culpa de los estudiantes, puede presentarse por niveles altos en sus expectativas académicas no cumplidas y al desprecio personal por errores o injusticias cometidas en el presente y el pasado. La no aceptación de un ser personal falible, que puede cometer errores o equivocarse constantemente, puede interferir como lo señala Ellis ${ }^{(3)}$, en los diferentes ámbitos de la vida que menguan la producción y la felicidad personal.

La irracionalidad de los sentimientos de culpa puede llevar a los alumnos a creer que no son dignos como personas, por los errores cometidos y pueden autoperpetuar una conducta que confirme posteriormente su poca validez o dignidad, cometiendo nuevos errores para validar sus creencias irracionales; y como lo señala Riso (1) sobre la autoperpetuación esquemática y profecías autorrealizadas en las personas que obedecen a un esquema o creencia irracional que lo van reforzado y confirmando inconscientemente a través de sus cogniciones, emociones y conducta. Otra consecuencia que puede tener una creencia irracional como el sentimiento de culpa es lo que manifiesta Ellis y McLaren (9), quien refiere que las creencias irracionales a menudo dan como resultado preocupación y sentimientos de culpa hacia sí mismos.

Por otro lado, la creencia irracional de grado de independencia, aparece con un predominio alto en el grupo de estudiantes de sexo femenino y no varones, resaltando está característica en la población estudiada según sexo. Esta es definida por Díaz ${ }^{(8)}$ citado por Aguinaga ${ }^{(6)}$ (2012) como la aceptación de Independencia en las decisiones y también en las consecuencias de las acciones. Es así, como podemos caracterizar a las alumnas con un alto grado de actitud de autonomía ante las causas externas.

En cuanto a los factores de las creencias irracionales en un nivel alto, presentes según su ciclo vital de desarrollo en los alumnos de la escuela de Derecho, se determinó que las creencias irracionales en el grupo de adolescentes están dadas por los sentimientos de culpa y en la etapa de adultez temprana, por creencias irracionales de sentimientos de culpa y grado de independencia. Craig ${ }^{(10)}$ aclara sobre la predominancia exclusiva de esta edad y el sentimiento de culpa; como la búsqueda en el adolescente de su identidad e in- dependencia total, lo que muchas veces lo llevará a confrontarse con figuras de autoridad, que son el reflejo de las normas sociales; que, al no cumplirlas cabalmente, se vuelven imperativos llevando a experimentar sentimientos de autorreproches y desvaloración de su propio ego.

Por otro lado, el sentimiento de culpa también está reflejado en los estudiantes del grupo de adultez temprana, como una manifestación de culpas por errores e injusticias, que ellos observan como consecuencias graves de sus actos; esta creencia irracional, está dada junto al grado de Independencia, manifestado como un factor alto de irracionalidad. Craig ${ }^{(10)}$, menciona que casi todos los adultos jóvenes, son más fuertes, saludables y fértiles de lo que fueron o llegarán a ser. Tienen más vigor y han definido una identidad y unas pautas sexuales que han de durar toda la vida.

Por último, se llegó a determinar que las creencias irracionales en un nivel alto, según la presencia de trabajo, es significativo en las creencias irracionales de sentimiento de culpa y grado de independencia. Así como, el nivel alto de creencias irracionales en los alumnos que no trabajan, solo se observa en la creencia irracional de sentimientos de culpa.

Evidenciándose que el sentimiento de culpa es la creencia irracional que más predominancia tiene en todos los grupos estudiados de esta investigación, lo que está dado también en los alumnos que no trabajan como una consecuencia de deberes en las demandas externas de un ambiente que privilegia el trabajo como un estatus especial en la jerarquía sociocultural. Llerena ${ }^{(11)}$, refiere que las creencias irracionales se pueden identificar como exigencias absolutistas y rígidas, expresadas como "debo de", "necesito", "tengo que", es así como los alumnos pueden manifestar también demandas para poder trabajar como una obligación en mejora de un estatus social. En cambio, los alumnos que trabajan muestran creencias irracionales de sentimientos de culpa y grado de independencia; al hacerse autorreproches por errores personales irracionales, manifiestan un grado de independencia irracional como consecuencia de tener un cierto nivel de autonomía que le proporciona el trabajo y que lleva a distorsionar la realidad a través de un egocentrismo y personalización de las causas externas.

Se pudo concluir que lo sentimientos de culpa y grado de independencia son las creencias irracionales con mayor prevalencia estudiantes de la Escuela de Derecho de una universidad particular de Chiclayo, donde en el sexo femenino y en la etapa de la adultez temprana se presentan con mayor significancia. Es necesario establecer estrategias de intervención para asistir a la población estudiantil en riesgo de creencias irracionales. 
Conflictos de interés: ninguno.

Fuentes de financiamiento: autofinanciado.

\section{REFERENCIAS BIBLIOGRÁFICAS}

1. Riso W. Terapia Cognitiva. Madrid: Lunwerg Editores; 2009

2. Antony Clark D, Temkin Beck A, Brad AA. Fundamentos científicos de la terapia cognitiva y la terapia de la depresión. New York: Editorial John Wiley \& Sons; 1999.

3. Ellis A, Dryden W. Práctica de Terapia Racional Emotiva. Bilbao, España: Descleé; 1989

4. López Manjarres E, Galarza Zurita GE. Ideas Irracionales y su influencia en las conductas agresivas de los estudiantes de los primeros cursos de bachillerato en los Colegios Fiscales Urbanos del cantón Pelileo de la Provincia de Tungurahua. [Tesis en internet]: Universidad Técnica de Ambato; 2015. Disponible en: https://repositorio.uta.edu.ec/jspui/bitstream/123456789/9319/1/L\%C3\%B3pez\%20Manjarr\%C3\%A9s\%2C\%20 Evelin\%20lsabel.pdf

5. Shorkey C, Whiteman V. Development of the Rational Behavior Inventory: Initial validity and reliability. United States: Educational and Psychological Measurement. 1977, 37(2): 527-34. https://doi. org/10.1177/001316447703700232

6. Aguinaga Alvarez A. Creencias irracionales y conductas parentales en madres víctimas de violencia infligida por la pareja. Rev Psicol Hered. 2012;6 (1-2): 1-11.

7. Huerta Hernández N. Inteligencia e ideas irracionales en estudiantes universitarios. [Tesis] [Mexico]: Universidad Iberoamericana Puebla; 2012

8. Díaz C. Diferencias en las creencias irracionales de un grupo de internos varones en función al delito cometido recluidos en un centro penitenciario de la ciudad de Huacho. [Tesis]. [Perú]: Universidad de San Martín de Porres; 2002.

9. Ellis A, Maclaren C. Las Relaciones con los Demás. Barcelona: Oceáno Ambar; 2004

10. Craig G. Desarrollo psicológico. México: Pretince Hall; 1997

11. Llerena Espezúa X. Salud psicológica en la teoría de Albert Ellis. Rev Psicol Arequipa 2015; 5(2):158-75 